\title{
Elevation of Blood Plasminogen Activator Inhibitor Level in Patients With Cerebral Venous Thrombosis
}

\author{
Suzan Bindal ${ }^{\mathrm{a}}$, Ozlem Coskun ${ }^{\mathrm{b}}$, Bulent Aliogluc ${ }^{\mathrm{c}}$, Levent E. Inan ${ }^{\mathrm{d}}$, \\ Burc Esra Sahine, g, Ruhsen Ocal ${ }^{\mathrm{f}}$
}

\begin{abstract}
Background: Cerebral venous thrombosis (CVT) accounts for $0.5 \%$ of cerebrovascular diseases (CVDs). A prothrombotic state can be demonstrated in $34 \%$ of the affected patients, while $22 \%$ of them had a genetic prothrombotic tendency. The main action of plasminogen activator inhibitor (PAI-1) is to regulate the fibrinolytic system. PAI-1 deficiency is associated with abnormal bleeding.
\end{abstract}

Methods: A total of 20 patients were confirmed to have CVT with brain magnetic resonance imaging (MRI) and MR venography, and 26 healthy controls were enrolled in this prospective study.

Results: PAI levels were studied in 20 patients who sustained a CVT attack and 26 healthy controls. PAI-1 levels were compared between patients with acute and chronic CVT as well. The mean PAI-1 levels in the healthy controls and patient group were 13.32 (4.58 - 47.72) and 40.66 (8.75 - 146.88), respectively. There was a significant difference between both groups with respect to PAI-1 level, with the patient group having a higher PAI-1 level. No significant differences in blood PAI-1 levels were found between acute and chronic CVT.

Conclusions: Our study demonstrated that PAI-1 level is a significant risk factor for acute and chronic CVT. In future, reducing PAI-1 levels may determine the treatment approach in CVT patients.

Keywords: Sinus vein thrombosis; Plasminogen activator inhibitor; Cerebrovascular diseases; Fibrinolytic system

Manuscript submitted March 11, 2018, accepted April 16, 2018

${ }^{a}$ Turkish Medicines and Medical Devices Agency, Republic of Turkey Ministry of Health, Turkey

bPrivate doctor of neurology,Special Clinic, Ankara, Turkey

'Department of Pediatric Hematology, Ankara Research and Training Hospital, Turkey

${ }^{\mathrm{d}}$ Department of Neurology, Bozok University Faculty of Medicine, Turkey eDepartment of Neurology, Ahi Evran University Faculty of Medicine, Turkey fDepartment of Neurology, Baskent University Faculty of Medicine, Turkey 'Corresponding Author: Burc Esra Sahin, Department of Neurology, Ahi Evran University Faculty of Medicine, Bagbasi Mah. Sahir Kurutluoglu Cad. No: 100 Merkez, Kirsehir, Turkey. Email: besras11@yahoo.com

doi: https://doi.org/10.14740/jnr472w

\section{Introduction}

Thrombosis of cerebral veins and sinuses accounts for $0.5 \%$ of cerebrovascular diseases (CVDs) [1]. A prothrombotic state can be demonstrated in $34 \%$ of the affected patients, while $22 \%$ of them had a genetic prothrombotic tendency [2].

There is a continuous balance between coagulation and fibrinolysis in the vascular system. Coagulation causes formation of fibrin deposits that, together with thrombin, are degraded by plasmin, a proteolytic enzyme. Plasminogen, the precursor of plasmin, is converted into the latter by plasminogen activator. Plasminogen activator inhibitor (PAI-1) is the major inhibitor of this conversion [3]. PAI-1's main role is regulation of fibrinolytic system. The variable balance between fibrinolytic and procoagulant systems greatly contributes to the pathophysiology of thrombus formation [4]. Suring fibrinolysis plasminogen is converted to plasmin to lyse fibrin clot. This conversion is activated by urokinase plasminogen activator ( $\mathrm{u}-$ $\mathrm{PA}$ ) or tissue plasminogen activator (t-PA) release. PAI-1 rapidly inhibits u-PA and t-PAI-1 release [5].

PAI-1 deficiency in humans is related to abnormal bleeding events. This suggests that PAI- 1 has an important role in stabilization of hemostatic plug. Its excessive secretion may interfere with normal fibrin clearance mechanism on vessel wall, leading to pathological fibrin deposition and thrombotic events [6]. Impaired fibrinolysis has been related to increased PAI-1 level in more than $40 \%$ of patients with venous thromboembolism. Increased PAI-1 is also a predictor of future thrombotic events [7]. The relationship between cerebral venous thrombosis (CVT) and PAI-1 is yet to be determined.

In this study, we compared the levels of PAI-1between patients with CVT and control group.

\section{Patients and Methods}

In this study, we included 20 CVT patients, which we confirmed their diagnosis by using magnetic resonance imaging (MRI) and MR venography. All of the patients consecutively followed at our neurology department. Fifteen patients' diagnosis was chronic cerebral venous thrombosis (symptoms developed over a period greater than 30 days and up to 6 months patients included chronic phase of the disease) and five patients had acute stage of the disease (symptoms presented in an acute 
fashion and the symptoms appeared in less than $48 \mathrm{~h}$ ). The diagnosis of the disease was confirmed by imaging modalities in each patient. We compared the results of 26 healthy controls who were enrolled prospectively in our neurology clinic.

Patients who met the following criteria were excluded from this study: patients being younger than 18 or older than 85 years; using anticoagulant drugs; having an acute infection, oncological disease, renal failure, or liver failure.

The healthy control group consisted of age-matched patients having no systemic disease or using any medication.

Our study was conducted in compliance with Declaration of Helsinki and good clinical applications protocol. Ministry of Health Ankara Training and Research Hospital local ethics committee approved our study protocol. A voluntary written informed consent was obtained from patients and controls.

A detailed history was taken from each patient regarding the symptoms, medications used, and vascular risk factors (diabetes, hypertension, heart disease, hyperlipidemia, previous cerebrovascular accidents, and cigarette or alcohol abuse). Detailed physical and neurological examinations were performed. History taking and physical examination were both carried out by a single experienced neurologist.

CVT and control group patients were matched according to sex and age. We also checked all patients' (CVT group and control group) liver function tests, creatinine levels, and glucose levels. We also investigated other risk factors for patients of CVT (for example, inherited prothrombotic risk factors).

\section{Statistical analysis}

The study data were analyzed using SPSS for Windows 15 software package. The descriptive statistics were presented as mean \pm standard deviation for normally distributed data and median (min. - max.) for those that were non-normally distributed. The nominal variables were expressed as the number of cases and percentage $(\%)$.

The significance of the difference between group means was investigated with the Student's $t$-test, while the significance of the difference of group medians was analyzed using Mann-Whitney U test. A P value less than 0.05 was accepted statistically significant.

\section{Laboratory methods}

Peripheral blood samples were drawn from each patient following an overnight fasting period at 08:00 to 10:00 am. The samples were preserved at $-80^{\circ} \mathrm{C}$ in closed tubes. Serum PAI-1 levels were determined using commercial kits using the ELISA method.

\section{Results}

According to the pre-specified criteria, a total of 20 patients, of whom five were in the acute stage and 15 in the chronic stage, and 26 healthy controls were enrolled in this study.
The mean age of the patient group was 33.5 (20 - 57) years. The average age of the control group was 32.5 (30 - 36) years, with no statistical difference $(\mathrm{P}>0.05)$.

The patient group was consisted of 16 females and four males, while the control group consisted of 23 females and three males, totaling 20 and 26 subjects, respectively. The female/male ratio was 4 in the patient group, while the female/ male ratio was 7.66 in the control group (Table 1).

Detailed neurological examination at admission revealed abnormal findings in $55 \%$ of patients.

Neuroimaging techniques were also used in our study. Eleven $(55 \%)$ of 20 patients had abnormalities in brain MRI. MR venographies taken in 20 patients revealed an occlusion of a single sinus in seven patients and more than one sinus in 13 patients.

PAI-1 levels were studies in 20 patients, of whom five were in the acute stage and 15 in the chronic stage, and 26 healthy controls. In addition, PAI-1 levels were also compared between the acute stage and chronic stage within the patient group.

According to the Mann-Whitney U test, the control group had a mean PAI-1 level of 13.32 (4.58 - 47.72), while the healthy control group had a mean PAI-1 level of 40.66 (8.75 146.88) $(\mathrm{P}<0.05)$ (Fig. 1).

Mann-Whitney U test revealed a significant difference between the patient and control groups with respect to the PAI-1 levels $(\mathrm{P}<0.05)$. The patient group had a higher PAI-1 level. This result was verified by an ROC curve $(\mathrm{P}<0.05)$, confirming the predictive power of PAI- 1 .

The PAI-1 level was also compared within the patient groups and no significant difference was noted between the patients with acute or chronic stage in accordance with MannWhitney U test $(\mathrm{P}>0.05)$ (Fig. 2).

A cut-off level was determined using Youden index. The sensitivity and specificity were determined as $75 \%$ and $96 \%$, respectively.

\section{Discussion}

CVT is a rare form of CVDs. It accounts for $0.5 \%$ of all CVD cases [1]. With the advent of neuroimaging modalities, it has recently shown that CVT is more common than once thought, has a variable mode of onset and clinical course, and usually portends a favorable prognosis. Since the risk of death and severe morbidity fall below $10 \%$ with appropriate therapy, early diagnosis is of paramount importance [8].

Unlike arterial stroke, CVT is especially common among young persons and neonates [8]. The estimated incidence is 2 - 4 persons per million population, with $75 \%$ of them being women [9]. The mean age was $33.5(20-57)$ years in the patient group and $32.5(30-36)$ years in the control group of our study and there was no significant difference between both groups with respect to mean age $(\mathrm{P}>0.05$ in Mann-Whitney U test).

CVT is more prevalent in women compared to men, with a female/male ratio of $3: 1$ [2-10]. This difference in disease rate also applies for young adults, but it is not valid for children or 


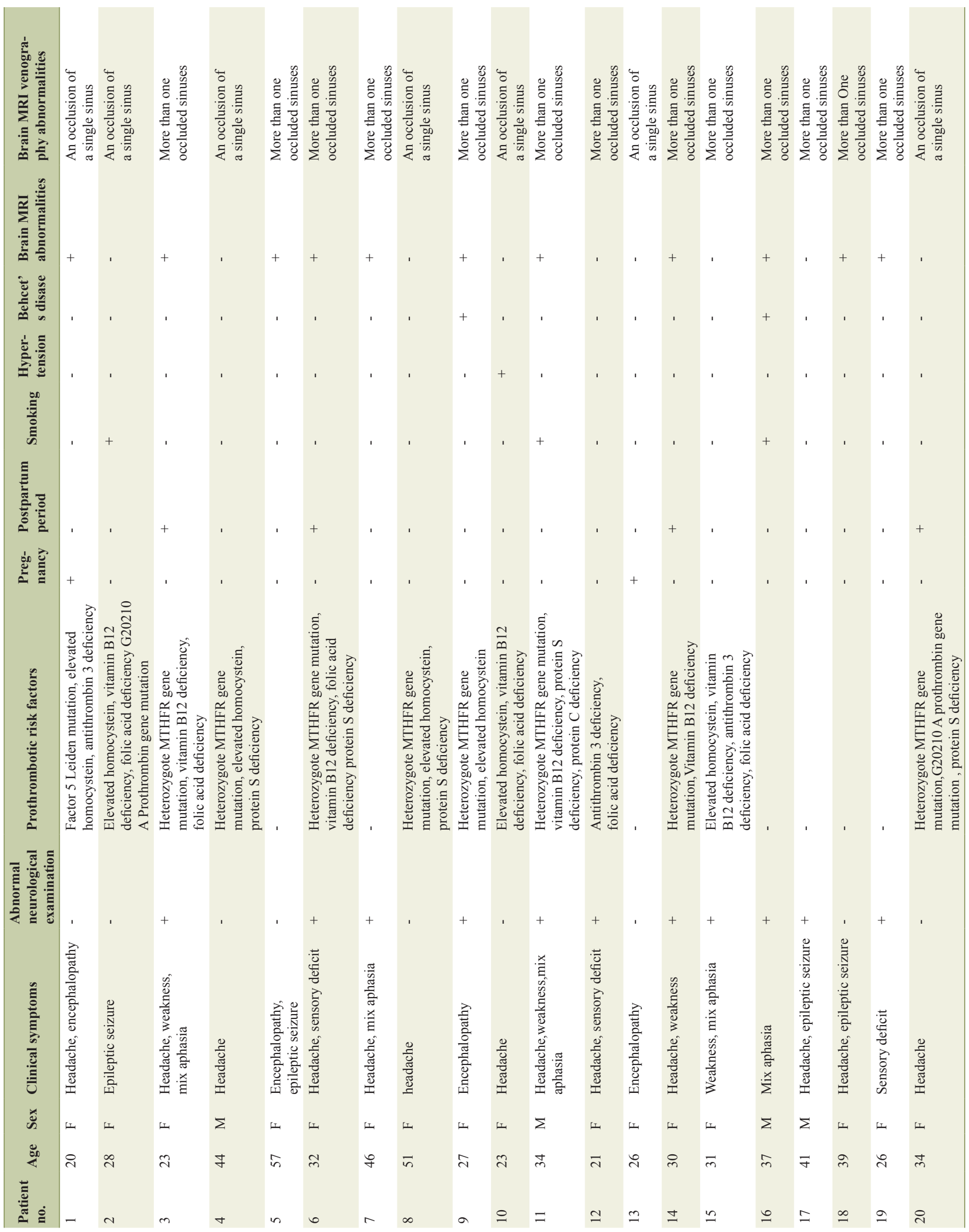




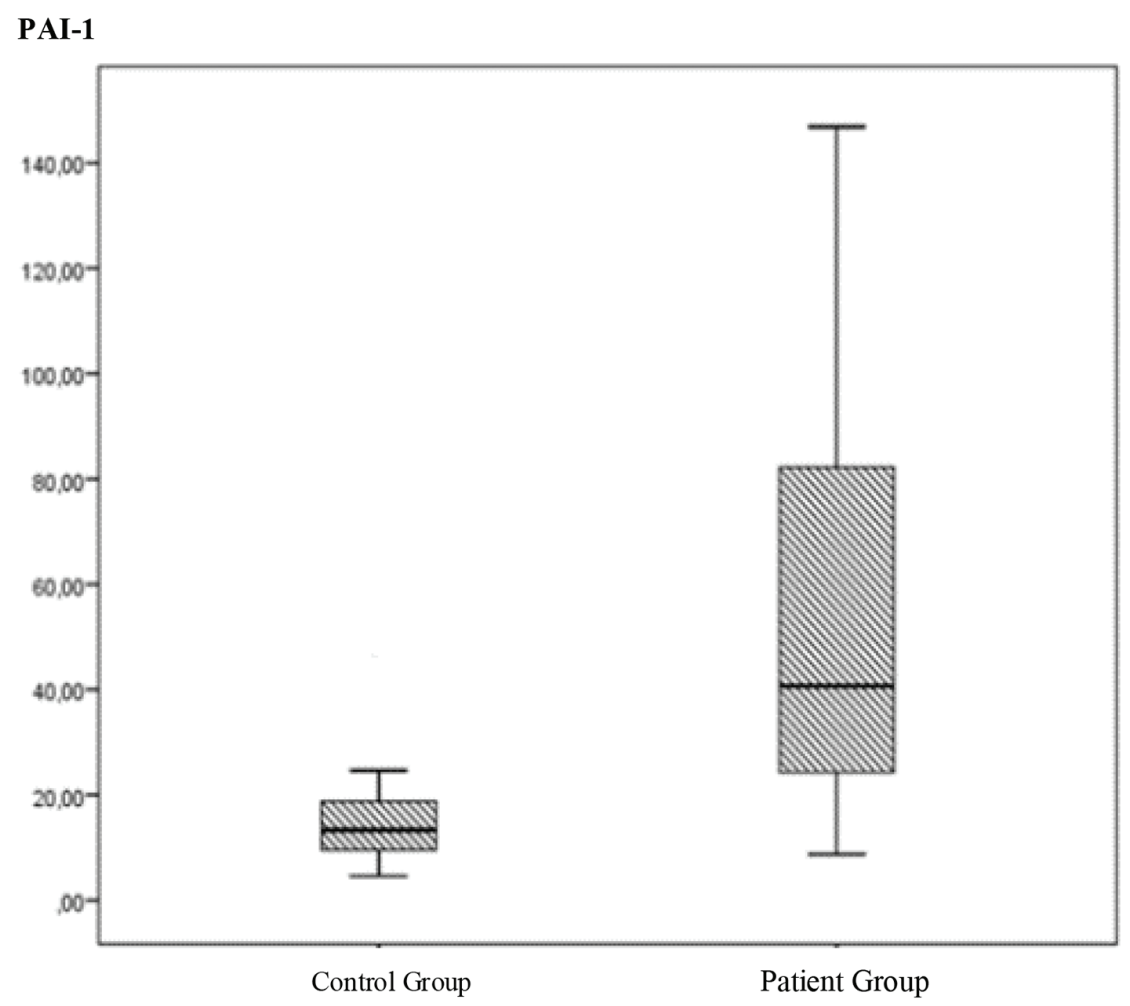

Figure 1. The comparation of the PAI levels between the control group and patient group.

\section{PAI-1}

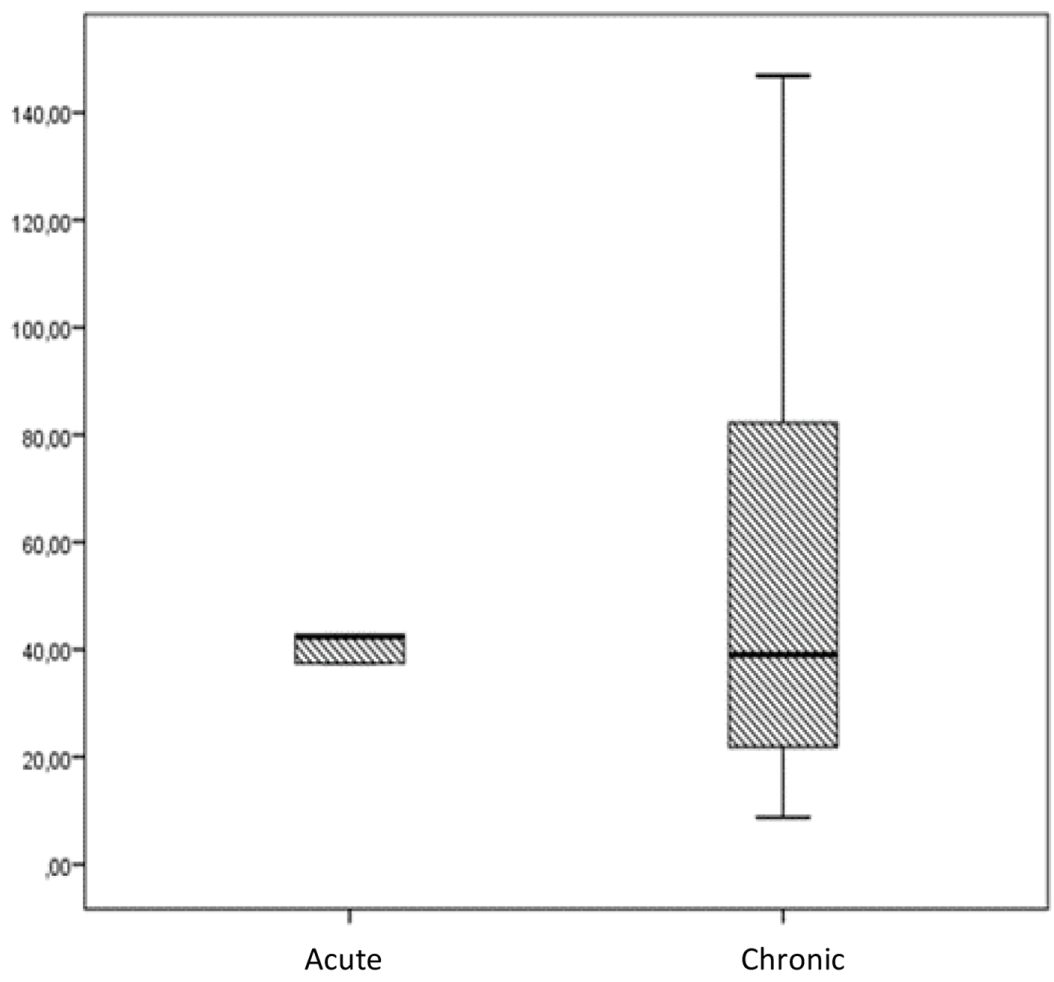

Figure 2. The comparation of PAI-I levels between acute and chronic stages of cerebral venous thrombosis (15 in the chronic phase and five in the acute phase). 
elderly [2-11]. In a 624-patient study where $75 \%$ of the participants were female, the mean age was 34 years in females and 42 years in males [10]. In our study, there were 16 females and four males in the patient group and 23 females and three males in the control group, totaling 20 and 26 subjects, respectively. The female/male ratio was $4: 1$. In the patient group, the mean age of females was 32.1 years, while it was 39 years in males.

Studies have demonstrated that $85 \%$ of affected patients have at least one risk factor, with prothrombotic risk factors being most common. Among affected patients, 34\% had a prothrombotic state and $22 \%$ had genetic background for the disease [2]. Among classical genetic prothrombotic causes, the activated protein $\mathrm{C}$ resistance caused by factor 5 Leiden mutation accounts for $10-20 \%$ of cases [12]. In our study, one of 18 patients had factor 5 Leiden mutation $(0.05 \%)$.

The relationship between CVT and hyperhomocysteinemia caused by a mutation in the methylene tetrahydrofolate reductase (MTHRF) gene is controversial [13-15]. However, hyperhomocysteinemia alone is an independent risk factor for CVT. It has been reported that hyperhomocysteinemia causes a four-fold increased risk of CVT in $27 \%$ of the affected patients [16]. We detected a heterozygote MTHFR gene mutation in eight of 17 patients $(47 \%)$. We also determined elevated homocystein levels in seven of 17 patients, vitamin B12 deficiency in seven of 19 patients, and folic acid deficiency in six patients.

The prevalence of G20210 A prothrombin gene mutation has been reported $11 \%$ among patients diagnose with CVT [14]. A total two of 17 patients had G20210 A prothrombin heterozygote gene mutation in our study $(0.1 \%)$

Among other hypercoagulability causes, protein $\mathrm{C}, \mathrm{S}$, and antithrombin 3 deficiencies, on the other hand, were reported to be quite rare [8]. We observed protein $\mathrm{S}$ deficiency in five of 16 patients and protein $\mathrm{C}$ deficiency in one patient. A total of three of 18 patients had antithrombin 3 deficiency in our study.

Postpartum period, pregnancy, and oral contraceptive use, which collectively represent estrogen excess and associated thrombosis tendency, were observed in a part of our patients and this is in agreement with the literature [17]. Two of 20 patients had pregnancy, and four had oral contraceptive use. Four patients were in the postpartum period (20\%).

From a practical standpoint, causes of peripheral arterial and venous thrombosis should also be taken into account in CVT. Our study showed that three of 20 patients had a history of smoking and one had hypertension. We have also taken into account predisposing conditions for arterial thrombosis. Seven of a total of 17 patients had elevations in total cholesterol and LDL cholesterol, while five had hypertriglyceridemia.

Behcet's disease is a vasculitis affecting both veins and arteries. CVT is one of the most common neurological complications of this syndrome. Two of our patients had Behcet's disease.

Nine of our patients had no remarkable feature in their past medical history (45\%).

Brain MRI and MR venography combination also including gradient echo T2-weighed sequences is considered as the most sensitive method for diagnosing a thrombus or an occluded dural sinus or vein [18-20]. We found a brain MR abnormality in $11(55 \%)$ of 20 patients. MR venography was performed in 20 patients and revealed an occlusion of a single sinus in sev- en patients and more than one occluded sinuses in 13 patients.

The main role of PAI-1 is regulation of the fibrinolytic system [4]. During fibrinolysis, plasminogen is converted to plasmin to lyse the fibrin clot. This conversion process is activated by release of $\mathrm{u}-\mathrm{PA}$ or t-PA. PAI-1 rapidly inhibits $\mathrm{u}-\mathrm{PA}$ and t-PAI-1 release [5].

In humans, PAI-1 deficiency is associated with abnormal bleeding. On the other hand, its excessive release may impair normal fibrin clearance mechanism on the vessel wall, resulting in pathological fibrin deposition and thrombotic events [6].

In more than $40 \%$ of patients with venous thromboembolism, an impaired fibrinolysis has been linked to increased PAI-1 levels. Increased PAI-1 levels are also predictor of future thrombotic events [7]. Elevated PAI-1 levels have been shown to cause both venous and arterial thrombosis [21]. Some studies have specifically sought for effects of PAI-1 on coronary artery disease and myocardial infarction [22].

In contrast to all the above information, no significant association was observed with PAI-1 genotype levels in adults and children with acute ischemic stroke, deep vein thrombosis and retinal thrombosis [23].

In conclusion, our study found that PAI-1 level is a risk factor in acute and chronic stage CVT. In future, reducing PAI1 levels may determine the treatment approach.

\section{References}

1. Bousser MG, Ferro JM. Cerebral venous thrombosis: an update. Lancet Neurol. 2007;6(2):162-170.

2. Ferro JM, Canhao P, Stam J, Bousser MG, Barinagarrementeria F, Investigators I. Prognosis of cerebral vein and dural sinus thrombosis: results of the International Study on Cerebral Vein and Dural Sinus Thrombosis (ISCVT). Stroke. 2004;35(3):664-670.

3. Sara F, Saydam G, Tuzun M, Kabalak T, Yilmaz C. Obezitede Trombosit Fonksiyonlari. Turkish Journal of Endocrinology and Metabolism. 2003;(Suppl. 2):69-72.

4. Tsantes AE, Nikolopoulos GK, Bagos PG, Bonovas S, Kopterides P, Vaiopoulos G. The effect of the plasminogen activator inhibitor- $14 \mathrm{G} / 5 \mathrm{G}$ polymorphism on the thrombotic risk. Thromb Res. 2008;122(6):736-742.

5. Stiko A, Hervio L, Loskutoff DJ. Plasminogen activator inhibitors. In: Colleman RW, Hirsh J, Marder VJ, Clowes AW, George JN, editors. Hemostasis and thrombosis: basic principles and clinical practice, 4th ed. Philadelphia: Pa: Lippincott Williams and Wilkins. 2001; p. 975-1002.

6. Koenig W. Haemostatic risk factors for cardiovascular diseases. Eur Heart J. 1998;19(Suppl C):C39-43.

7. Schulman S, Wiman B. The significance of hypofibrinolysis for the risk of recurrence of venous thromboembolism. Duration of Anticoagulation (DURAC) Trial Study Group. Thromb Haemost. 1996;75(4):607-611.

8. Masuhr F, Mehraein S, Einhaupl K. Cerebral venous and sinus thrombosis. J Neurol. 2004;251(1):11-23.

9. Khan M, Kamal AK, Wasay M. Controversies of treatment modalities for cerebral venous thrombosis. Stroke Res Treat. 2010;2010:956302.

10. Coutinho JM, Ferro JM, Canhao P, Barinagarrementeria 
F, Cantu C, Bousser MG, Stam J. Cerebral venous and sinus thrombosis in women. Stroke. 2009;40(7):23562361.

11. DeVeber G, Andrew M, Adams C, et al. Cerebralsinovenous thrombosis in children. N Engl J. 2001;Med $345: 417$.

12. Deschiens MA, Conard J, Horellou MH, Ameri A, Preter M, Chedru F, Samama MM, et al. Coagulation studies, factor V Leiden, and anticardiolipin antibodies in 40 cases of cerebral venous thrombosis. Stroke. 1996;27(10):17241730 .

13. Marjot T, Yadav S, Hasan N, Bentley P, Sharma P. Genes associated with adult cerebral venous thrombosis. Stroke. 2011;42(4):913-918.

14. Hillier CE, Collins PW, Bowen DJ, Bowley S, Wiles CM. Inherited prothrombotic risk factors and cerebral venous thrombosis. QJM. 1998;91(10):677-680.

15. Wysokinska EM, Wysokinski WE, Brown RD, Karnicki K, Gosk-Beirska I, Grill D, McBane RD, 2nd. Thrombophilia differences in cerebral venous sinus and lower extremity deep venous thrombosis. Neurology. 2008;70(8):627-633.

16. Martinelli I, Battaglioli T, Pedotti P, Cattaneo M, Mannucci PM. Hyperhomocysteinemia in cerebral vein thrombosis. Blood. 2003;102(4):1363-1366.
17. Agostoni E, Aliprandi A, Longoni M. Cerebral venous thrombosis. Expert Rev Neurother. 2009;9(4):553-564.

18. Chu K, Kang DW, Yoon BW, Roh JK. Diffusion-weighted magnetic resonance in cerebral venous thrombosis. Arch Neurol. 2001;58(10):1569-1576.

19. Dormont D, Anxionnat R, Evrard S, Louaille C, Chiras J, Marsault C. MRI in cerebral venous thrombosis. J Neuroradiol. 1994;21(2):81-99.

20. Rizzo L, Crasto SG, Ruda R, Gallo G, Tola E, Garabello D, De Lucchi R. Cerebral venous thrombosis: role of CT, MRI and MRA in the emergency setting. Radiol Med. 2010;115(2):313-325.

21. Eitzman DT, et al. Plasminogen activator inhibitor 1. Textbook of Coronary Thrombosis and Thrombolysis. In: Becker RC, (Ed), Norwell, MA: Kluwer Academic Publishing. 1997.

22. Kohler HP, Grant PJ. Plasminogen-activator inhibitor type 1 and coronary artery disease. N Engl J Med. 2000;342(24):1792-1801.

23. Ringelstein M, Jung A, Berger K, Stoll M, Madlener K, Klotzsch C, Schlachetzki F, et al. Promotor polymorphisms of plasminogen activator inhibitor-1 and other thrombophilic genotypes in cerebral venous thrombosis: a case-control study in adults. J Neurol. 2012;259(11):2287-2292. 